\title{
An Optimized Player Taxonomy Model for Mobile MMORPGs with Millions of Users
}

\author{
Fang You, ${ }^{1}$ Jianping Liu, ${ }^{2}$ Xinjian Guan, ${ }^{2}$ Jianmin Wang, ${ }^{2}$ Zibin Zheng, ${ }^{3}$ and Peter Tam ${ }^{4}$ \\ ${ }^{1}$ School of Communication and Design, Sun Yat-Sen University, Guang Zhou 510006, China \\ ${ }^{2}$ School of Information Science and Technology, Sun Yat-Sen University, Guang Zhou 510006, China \\ ${ }^{3}$ Department of Computer Science and Engineering, The Chinese University of Hong Kong, Hong Kong \\ ${ }^{4}$ Gameislive Corporation, 5/f, 4 Victory Avenue, Kowloon, Hong Kong
}

Correspondence should be addressed to Jianmin Wang, mcswjm@mail.sysu.edu.cn

Received 7 June 2011; Accepted 26 September 2011

Academic Editor: Anthony Savidis

Copyright ( 2011 Fang You et al. This is an open access article distributed under the Creative Commons Attribution License, which permits unrestricted use, distribution, and reproduction in any medium, provided the original work is properly cited.

\begin{abstract}
Massively multiplayer online role-playing games (MMORPGs) have great potential as sites for research within the social and human-computer interaction. In the MMORPGs, a stability player taxonomy model is very important for game design. It helps to balance different types of players and improve business strategy of the game. The players in mobile MMORPGs are also connected with social networks; many studies only use the player's own attributes statistics or questionnaire survey method to predict player taxonomy, so lots of social network relations' information will be lost. In this paper, by analyzing the impacts of player's social network, commercial operating data from mobile MMORPGs is used to establish our player taxonomy model (SN model). From the model results, social network-related information in mobile MMORPGs will be considered as important factors to pose this optimized player taxonomy model. As experimental results showed, compared with another player taxonomy model (RA model), our proposed player taxonomy model can achieve good results: classification is more stable.
\end{abstract}

\section{Introduction}

With the widely use of cellular phones and rapid development of mobile network, MMORPGs on mobile are more and more popular. In mobile MMORPGs, players can be divided into different types on the basis of their attributes. Analysis of player taxonomy model could get the precise proportion and current situation of different types of players. It will help game operator to balance the proportion among different types of players and to optimize the game design. On the topic of player taxonomy study, Bartle [1] proposed four-type player taxonomy for MUDs: Achiever, Explorer, Socializer, and Killer. But this study was only based on interview and questionnaires to collect players' attributes data. The result was easily influenced by players' subjective attitude (Carrington et al. [2]). By analyzing mobile game operating data in our previous research [3] we built up an RA taxonomy model on the basis of player's own attributes. This model divides all the player behaviors into interaction and action and uses two motivations (Relationship and
Achievement) to explain these behaviors. Using the Relationship and Achievement scores, players are classified into 3 types.

In MMORPGs, recent studies show that MMORPGs have great potential as sites for research within the social and human-computer interaction. Yee $[4,5]$ and Castronova [6] find that players are not just playing a game together; moreover, their associations present as a social group. Many MMORPGs provide virtual environments to facilitate socialization and interactions among groups. Ducheneaut et al. [79] also propose that sociability should be considered in game design. By analyzing game design, Jakobson and Taylor [10] consider that typical virtual social network relationship exists in MMORPGs which means that virtual world in the games has its social structure. In continuous study, Ducheneaut et al. [11] propose that the number of social guild members determines the existence of the guild. In 2008, Bo et al. [12] find that different social guilds would choose players suitable to the guilds. Simultaneously, Bo et al. propose that social network could represent player taxonomy model. 
From the previous study, we assume that social network reflect players' motivation in the game process which means social network is also a considerable factor to build player taxonomy model. To study players' taxonomy model, we use commercial game "Empire Online (http://www.lakoo.cn/ $\mathrm{d} / \mathrm{g} /$ )" operating data to conduct quantitative analysis on players' own attribute and social network relations. We also propose a new player taxonomy model (SN model). This model will research both players' own attribute and their social network relations. To establish SN model, we analyze how players' own attribute affect player's taxonomy, and also calculate the weights in the taxonomy which are affected by different social relations. The data from a real commercial game are less affected by subjective factors; the model result is more reliable and suggestive to the game design.

The result of this paper is organized as follows: in Section 2, the impacts on players' taxonomy by social network relations would be analyzed. In Section 3, the taxonomy model will be introduced. In Section 4, a taxonomy model will be calculated by using commercial game operating data and will be compared with other taxonomy models. Finally, related results and future work will also be addressed.

\section{Social Networks in MMORPGs}

2.1. "Empire Online" and Social Group in MMORPGs. "Empire Online" is one of the most popular Turn-based commercial launched mobile MMORPGs in China. It runs on more than 800 types of mobile phones owned by a million subscribers since 2005. In the game, players first create an alter ego by choosing from four different race (e.g., east or Nordic) and six different character classes (e.g., mages or thieves). Once their character is built, players can begin questing in Kowloon. Kowloon is an extremely vast and richly detailed 2D environment. Players use weapons to attack monsters and wear armors to protect them. This allows them to earn "experience points" and reach higher "Levels" (70 is the current maximum level), acquiring powerful items and improving their abilities.

Meanwhile, players interact with the game and other players through an interface which closely resembles those of online game (Figure 1). Players communicate with each other by typing text in the text label at the right of the screen. There are some efficient communication channels available: one-to-one, group chat, and so on.

With the game's large subscriber, players must choose a specific server to play on. Each server can host about 20,000 players. Each server in the world of Kowloon is divided into four continents, and each is further subdivided into cities. Players can travel across these cities by using various props (e.g., flying sword) or on foot. Each race has a particular city which plays a role as transportation hub. Players can build an auction house, where players can trade objects on a market.

In the game, players can collect weapons, kill monsters, and PK with each other; they can also make friends and set up a family; most importantly, the players can create their own country and build up their own social relations.

In the game, players can make friends; they could add other players' name in the friend list. They could get

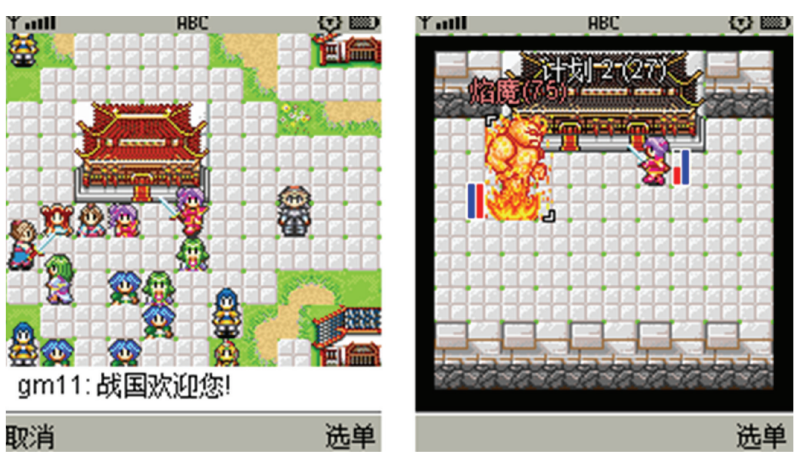

FIGURE 1: GUI interface of the "Empire Online" game.

married with players in opposite gender. When a new novice is not familiar with the rules of this game, he can also search the master or tutor to play with in the game. Especially, several players can set up their own country which composes of a king, several generals, and soldiers. Countries collect taxes from the members on regular; when another country invades, the king must summon members to defend his country.

From the above introduction, the "Empire Online" mobile game also has typical features in social network. In the game, a player will have lots of social relations. In our study, each social relation could be created as social group in theory. However, mobile MMORPGs is simpler, and the relationships of players consist of fewer relations (e.g., friend, family, country, apprentice, and trade). Therefore, we just analyze the social relations which have great influence on players' taxonomy. The relations like trade and apprentice will be ignored in our research.

2.2. Players' Social Relations. The social relations are various in MMORPGs. In general, players have friends, apprentices, and spouse as in the real world. As for most MMORPGs, guild is a special organization for players to communicate with each other [11] and to finish teamwork tasks. In our study, we defined the player set which is built up through specific relationships among players as "social group". For example, when a player has many friends, a few to tens, they will make up this particular player's friend network. The group in which players are friends is defined as "friend group". The group in which players are willing to spend money and time on country construction is defined as "country group". Three group type of social relation group in the game show as follows.

(i) Player to Player. The relationship between one player and another can be built by random relation such as friends, spouse, apprentices, and masters.

(ii) Player to Group. This kind of relationship can be set up by membership like labor unions, countries, and so forth.

(iii) Group to Group. The relationship can be established by competition and cooperation between communities. 
On the basis player-oriented relation, the relations like Player to Player as well as Player to Group are of great value. However, Group to Group relation mostly reflects the connection and communication between groups, which has little impact on player taxonomy model. Therefore, we will mainly research the first two relations definition, because these two relations form a virtual social network, which shows players' interests and behaviors. In the "Empire Online" game, we define three main relation groups which have major impact on player taxonomy.

(i) Friend Group. In MMORPGs, The friend relation is the most widely relationship in the game. Basically, all MMORPGs may have this relation mechanism. Players can have many friends as they play in the game. They gather together to accomplish their mission, kill monster, and PK with each other. Friend group is our most concerned groups. This social group belongs to the Player to Player group type.

(ii) Couple Group. Couple relation is a very special social relationship. When two opposite gender players are interested in each other, they will set up a family in the game. However, about 30\% players who already have family will have two or more spouses by our survey. The couple relation is more stable than the friend relation. In our study, when the player has couple group and friend group, we will regard Couple group as an independent group to be analyzed.

(iii) Country Group. Players in "Empire Online" could create countries. In each country, there will be one king, several generals, and many soldiers. The citizens in the country could donate their money or resource to the king for construction. Also, citizens could use the facilities in the country to upgrade their weapons and exchange resources.

2.3. Relation Network Analysis in Game. Lots of studies allow for direct empirical testing of long-stranding hypotheses on social network such as the hypothesis of weak ties (Granovetter [13]) and the hypothesis of social balance (Harary [14], Doreian and Mrvar [15]). In this part, we also build social network for each group using the methods "bridging" tie and "bonding" tie, which were proposed by Ducheneaut et al. $[8,11]$. The "brigdin" tie that players are connected to each other if they are found at the same time, irrespective of their game location (this tie is proportional to calculate the overlap time between the two players). It connects players who have the opportunity to interact by using the "game channel" and who are listed in the "friend list" window each time when a player logs in. This method is very suitable to create Friend Group and Couple Group. The "bonding" ties, in addition to the players' overlap time, the same location between players are also considered. Players can spend time together and group with other players to make mission and trade items. The country social group is very suitable for this method.

In order to demonstrate the social network in the social group, a specified relation group (Country C1) in "Empire Online" is selected to create social network by "bonding" tie

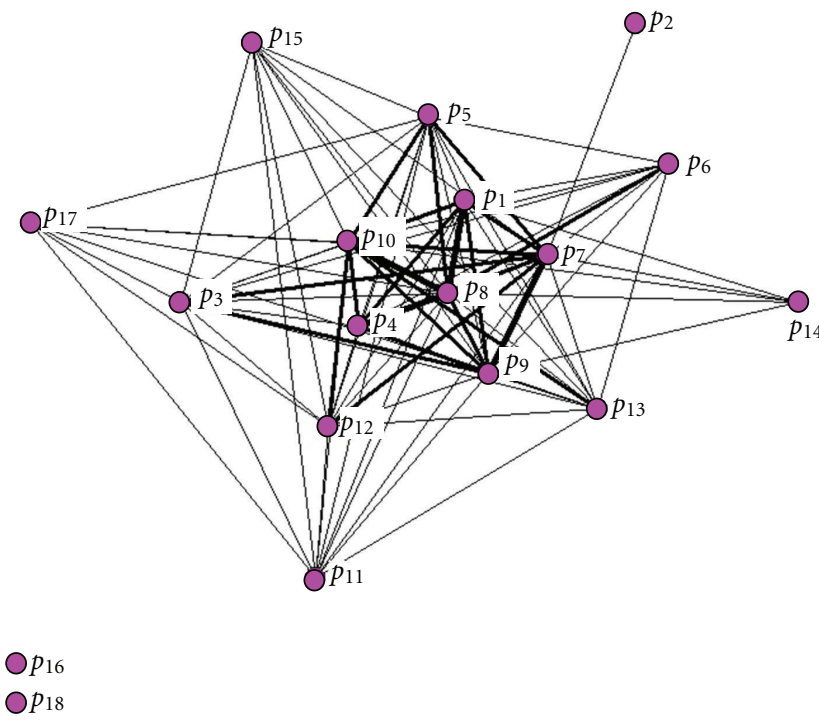

Figure 2: Country C1 relation network.

method. The $C 1$ group is a country relation in the game. The figure is shown as follows, and $p 1$ stands for player no. 1. From Figure 2, country group consists of 18 players. Because the overlap online time is short, p16 and p18 are assumed as missing players or new comers. Country social relation is defined as a social network in which players' relations show as connections. The individual player without connection is defined as external member of the country. The thicker line indicates that the players have more overlap time between each other. It means the stronger connection exists between these two players. The distance between nodes has no practical significance. Research found that many players do not contact other players although they are in a same social group. To reduce the impacts of these external players, we propose the concepts of Core-Relation group and CorePlayer.

(i) Core-Player. Some players are more active in the social groups. Their interaction is much more than others, which means they contribute more to the social groups. They could represent the main features of social group.

(ii) Core-Relation Group. All Core-Players form the CoreRelation Group. Core-Relation Group is tied strongly. It is the foundation of the whole social group

In our model, we select players whose overlap time is the top $30 \%$ of the players in the social group as core-players. In the $C 1$ country group, $p 1, p 4, p 7, p 8, p 9$, and $p 10$ are coreplayers, and they form a Core-Relation Group.

The Core-Players could stand for Core-Relation Group; the Core-Relation Group represents this whole relation group. We can quantify and analyze the social group's feature through these definitions. The overlap time between players could be used to analyze the social degree between players. Further, by analyzing the changes of this social group in specific moment, we could verify the impacts on players taxonomy made by the relation group. 
TABLE 1: Attributes of active players.

\begin{tabular}{lll}
\hline Category & Attributes & Description \\
\hline Relationship & Rank & Position in groups \\
& Friend & number of friends \\
& Family & Have wife or husband? \\
Achievement & KillCount & number of kill monster \\
& SumSkillLevel & Power of player skills \\
& Level & Level in game \\
\hline
\end{tabular}

\section{The Optimized Player Taxonomy Model on the Basis of Social Network: SN Model}

3.1. Player's Own Attributes. In our previous studies [3] proposed RA model in which we used players' attributes to analyze player taxonomy. Players' attributes have different scores; all the players' motivations are divided into two types by these attributes: Relationship and Achievement (Table 1). By comparing the scores in relationship motivations and achievement motivations, we can divide players into three categories: Socialize, Achiever, and Moderator. Specific types are described below.

(i) Socialize. This type measures the desire on communication and cooperation with other players in the game. Players' interaction activities and related attributes are used to evaluate the score of this motivation (e.g., The number of friends, the frequency of talking and the number of joining a war). The score of relative social behaviors could be measured in this section.

(ii) Moderator. It contains features from both relationship type and achievement type. Players not only like self-realization, but also like socialization and interaction. So, they are moderate in player groups.

(iii) Achiever. This type represents the players' goals and self-realization desire. It represents a player has strong game-related purposes (e.g., gathering money, upgrading, searching treasure, etc.). It can be measured by the player's self-related actions and attributes, such as weapon, skill level, and attack power.

3.2. SN Model. Theories of person-environment fit (Schneider [16]) and attraction-selection-attrition theory (Schneider et al. [17]) both agree that player taxonomy reflect on social networks. Williams et al. [18] propose that population of online players are a representative sample of real-world societies. Several recent researches show that human behavior on collective level is remarkably robust and statistical differences of real-world communities and game societies are often marginal (Johnson et al. [19] and Szell and Thurner [20]). Players have the possibility to create some groups in the game. The dynamics and interaction can be investigated readily in the data. This means that social network has a great impact on players taxonomy. In order to get a better taxonomy model, players' social network should be considered as a factor to analyze and quantify the impacts of social relations.

Through game analysis, we should consider not only the players' attributes, but also social relations. In player taxonomy, players' own attributes and social groups between the players have different weights. We should firstly calculate the own attributes score and then the relation score. In the first step, we have a detailed analysis in previous study [3]. The calculation has also been elaborated in the previous research. In the $\mathrm{SN}$ model, we define $N_{i}$ as the attribute score with player $i$ calculated by RA model. $p_{i}$ is the player set with player $i$.

In the second part, we should identify the Core-Relation Group and Core-Player and then use (1) to calculate the relation score

$$
R_{i j}=\frac{\sum_{l=1}^{n} c_{i l}^{j}}{n} .
$$

Here, $c_{i l}^{j}$ : Core-Player attributer score with Core-Player $i$ between Core-Player $l$ in social group $j, j=0$ means the Core-Relation Group and $j$ does not exist. $n$ : The number of Core-Players in social group $j . R_{i j}$ : The relation score with Core-Player $i$.

On the basis the formulas above, we propose players taxonomy model based on social network ( $\mathrm{SN})$. In this model, we use players' own attributes and social network to measure players' motivations to play MMORPGs. As shown in (2), we can quantify calculate the value of player taxonomy score

$$
K_{i}=\left(1-\sum_{j=1}^{k} w_{j}\right) N_{i}+\sum_{j=1}^{k} w_{j} R_{i j}
$$

Here, $w_{i}$ : The weight of social group $j . k$ : The number of social groups in model. $K_{i}$ : The player taxonomy score of player $i$. $N_{i}$ : The attributer score of player $i$.

In the model, the social group may have unlimited number in theory. Therefore, we could find social relations which can mostly reflect player taxonomy. The SN model is composed of RA model and social network factor. In our study, we mainly study the impact of social networks. And then we analyze how to influence the player taxonomy.

However, there are still several factors should be supplementary in the model.

Player has infinite number of social relations theoretically. But according to the abstractive categories of social relations, the major relations affected the players' type are Player-to-Player and Player-to-Group. In (2), we ignore the Group-to-Group relations.

In the MMORPGs, there are many inclusive players among social groups. For example, players $p 1, p 2, p 3, p 4$, $p 5, p 6$, and $p 7$ are friends, and they build a friend group. Simultaneously, players $p 1, p 5, p 6, p 7, p 8, p 9, p 10$, and $p 11$ come from the same country; they build a country group. $p 5$, $p 6$, and $p 7$ are inclusive players of these two different social groups. There could be two situations. First, these inclusive players do not belong to Core-Relations Group, which means these inclusive players could be omitted in relation score 


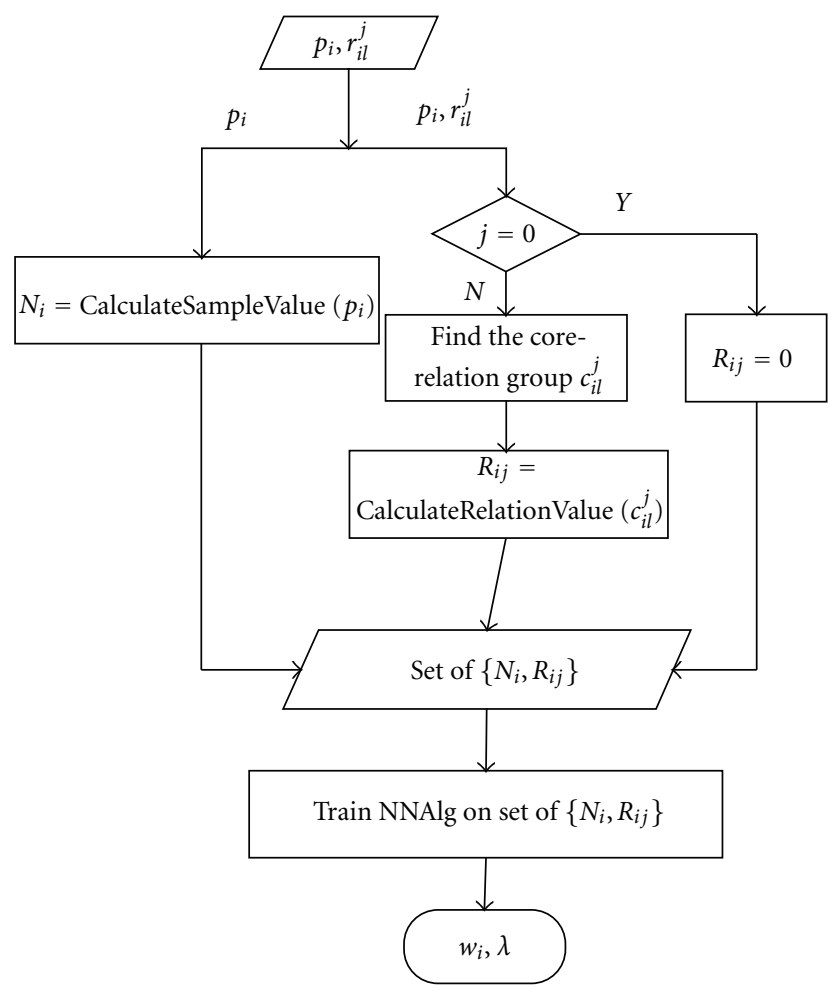

FIgURE 3: SN model algorithm flow diagram.

calculation. Second, if these inclusive players belong to CoreRelations Group, they have their own weights in relation score of different groups in (1). In conclusion, inclusive players have no fundamental effect on relation score calculation.

For more details, we will introduce the algorithms and the establishment of SN model in next section.

3.3. Model Calculation Process. In this section, the component of model will be introduced, and then, three main algorithms will be described in detail. In the taxonomy model process based on social group, player set $p_{i}$ and player attributer score set $r_{i l}^{j}$ as input data. The value of player own attributes $N_{i}$ will be calculated by RA model firstly. After judging whether player has social group, calculate the social group value of player. If social group does not exist, the social groups score $R_{i j}=0$, else find the Core-Player attributer score $c_{i l}^{j}$ in the $r_{i l}^{j}$. We can calculate the social group score $R_{i j}$ by the function CalculateRelationValue $\left(c_{i l}^{j}\right)$. Through the above process of calculation, players attribute and relations score set of $\left\{N_{i}, R_{i j}\right\}$ can be obtained. Finally, by training this score set with NNAlg, the NNAlg is the perception algorithm in the neural network; the ability of pattern recognition can be acquired by supervised learning. After training this data, the weight $w_{j}$ with social group of $j$ will be achieved. The process is shown in Figure 3.

3.4. Player Core-Relation Group Calculation. In the social network, players can often be categorized into groups on the basis of their interests. Players who have similar interests

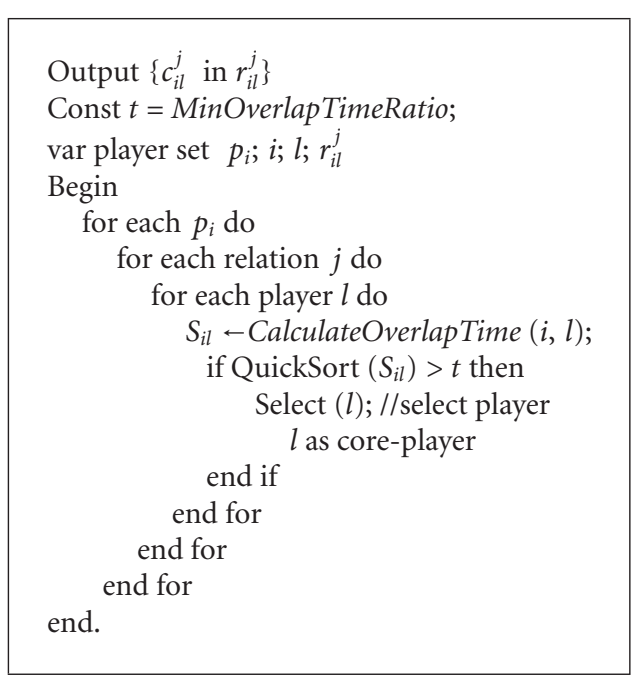

Algorithm 1: Find the core relation group.

become friends. Most players have a variety of relation groups. Some groups may be enormous. For example, the country groups may have more than 100 players. Meanwhile, there may be only two players in a family group.

In the investigation, many relation groups have formed small Core-Relation Groups with several Core-Players. The average frequency of interaction in Core-Relation Groups is more than the whole group. Core-relation Group can be representative of the group's information. We can quantitatively calculate the impacts on players by Core-Relation Group. Ducheneaut's "bonding" tie [9] approaches are used to find out the Core-Relation Groups in the groups. We propose Algorithm 1 to find the Core-Relation Groups. The algorithm outline shows as follows. The inputs of the algorithm are a player set $p_{i}, r_{i l}^{j}$ in the Algorithm 1. $r_{i l}^{j}$ is all players attributer score set in the social group $j$ with player $i$ between player $l$. $c_{i l}^{j}$ is Core-Player attributer score set. We need to find the $c_{i l}^{j}$ in the $r_{i l}^{j}$. The $S_{i l}$ is the score of the overlap time between the player $i$ and player $l$; in the algorithm, we iteratively calculate the overlap online time between player $i$ and player $l$ by function CalculateOverlapTime $(i, l)$, and then, Core-Player players could be selected when $S_{i l}$ is larger than threshold in relation group. Through this function, the Core-Players set $c_{i l}^{j}$ will be obtained.

Using this algorithm, we can select the Core-Relation Group and Core-Players from the relation groups.

3.5. Player Own Attributes Score and Relation Score Calculate. For the purpose of this paper, we use Algorithm 1 to find the Core-Relation Group and use Algorithm 2 for quantitative calculation of both own attributes score and relation score. For relation score, we can calculate the average of CorePlayers attributes score as the relation score.

In the Algorithm 2, we iteratively use the function CalculateSampleValue $\left(p_{i}\right)$ to calculate player's own attribute score $N_{i}$ and then use function CalculateRelationValue $\left(c_{i l}^{j}\right)$ to get the relation score $R_{i j}$ (Algorithm 2). Using this algorithm, 


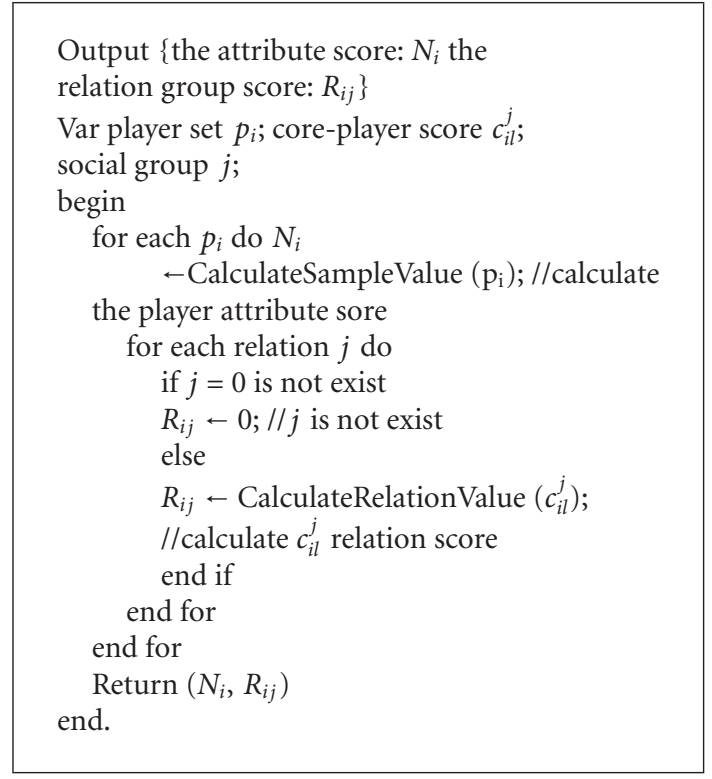

Algorithm 2: Calculate RA and Social group value.

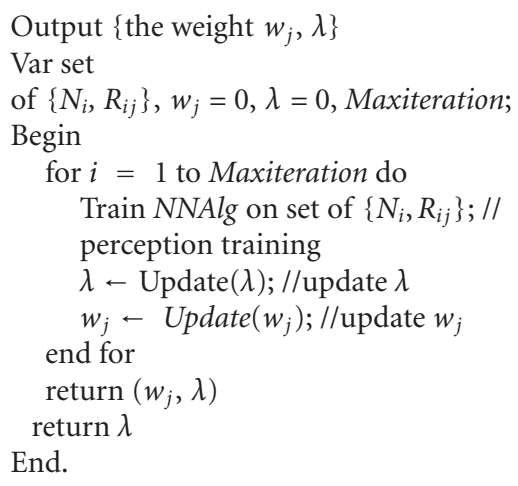

Algorithm 3: Calculate weights of categories $w_{j}$ and $\lambda=(1-$ $\left.\sum_{j=1}^{k} w_{j}\right)$.

we can get data set by players' own attributes $N_{i}$ and players' relations $R_{i j}$.

3.6. The Weight of Categories Calculate. In order to get precise data on how various factors affect players' taxonomy, we propose Algorithm 3 to calculate the weights. The inputs to the algorithm are data set of $\left\{N_{i}, R_{i j}\right\}$, initial weight set $w_{j}$ and $\lambda$, and the maximum number of iterations as Maxiteration. Using the perceptron model to train the data set, we can get the weight of all related factors. The perceptron model is a type of artificial neural network. It can be seen as the simplest kind of feed forward neural network. The output is the weight of all factors $w_{j}$ and $\lambda$. After normalization of the weight $w_{j}$ and $\lambda$, we can get the last percentage of all factors including attribute and relations.
TABLE 2: Active player's attributes score and weight with RA model.

\begin{tabular}{llcc}
\hline Category & Attributes & Average score & Weight \\
\hline Relationship & Rank & 1.61 & 0.38 \\
& Friend & 38.49 & 0.26 \\
& Family & 0.11 & 0.36 \\
Achievement & KillCount & 8390.6 & 0.36 \\
& SumSkillLevel & 10.2 & 0.29 \\
& Level & 43.33 & 0.35 \\
\hline
\end{tabular}

\section{Practice}

To verify the model, we applied it to a commercial mobile "Empire Online" game. In this section, the analyzed data came from a large-scale mobile online game (MMORPG) "Empire Online", which has more than 500,000 online players.

The score of the social relation in $\mathrm{SN}$ model is calculated by "bonding" tie approach. Players of overlap time data and login game frequency data will be available in this method. Some erosion of the players and inactive players cannot reflect the game situation very well. In order to make the player taxonomy model more accurate, the active players' data will be used to create the SN model.

The most active players are extracted from May to August in one service area in 2009. According to player online time and payment in the game, we select players who had accumulated more than three days' time a month for game in average for their avatars. In this way, we can make sure the player is active player. Finally, 4095 active players are selected.

By analyzing players' social groups, the relations can be divided into friends, countries, couple, and so on.

4.1. The Results of Experiment. According to "Empire Online" game, 6 representative players' attributes were selected by pretreatment and feature subset selection in Table 2 . Using variables clustering analysis and bivariate related analysis, 6 representative attributes were clustered into two categories.

By calculating the average of Core-Relation Group, the relation score can be obtained. The score of three relation categories are shown in Figure 4 . The $y$-axis is $R_{i j}$, which indicates the relation score of player in relation $j$; the $x$-axis is the player' identifier.

By calculating the average of these three social groups' relation score, the average score of Couple group is 0.833 . The average score of Friend group is 1.421 and of the country group is 2.257. It clearly shows that the score of social groups increases from couple groups to countries groups.

By calculating the value of player's own attributes score and social groups score, through the Algorithm 3, the weights of different factors are shown in Table 3.

From the results, it can be seen that player's own attributes have major effect on taxonomy model. However, the influence of the social groups cannot be ignored. The weight of three main social groups has almost nearly $1 / 3$ weight in the model. It can be concluded that social network has important impacts on player's taxonomy. 


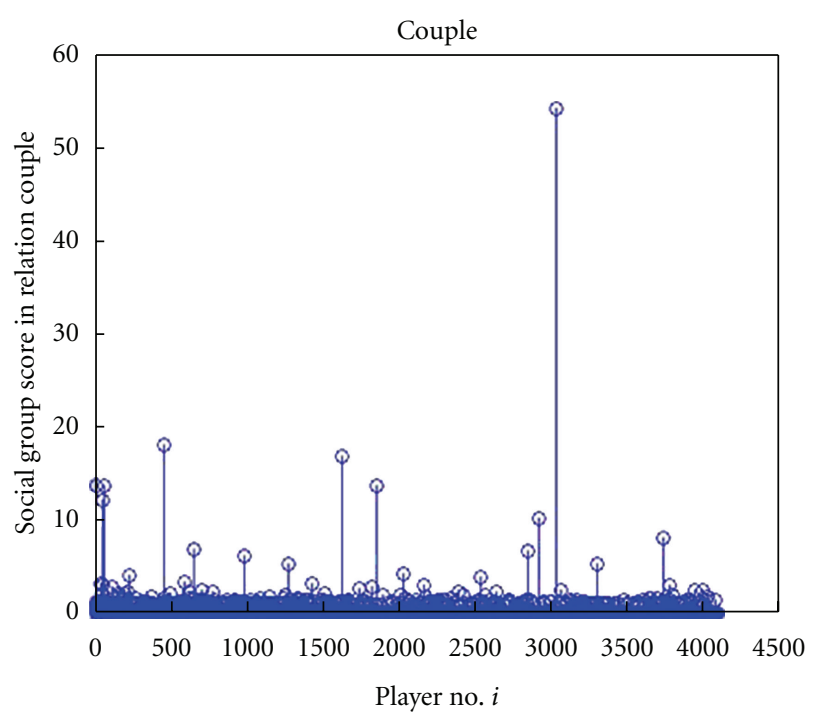

(a) Couple

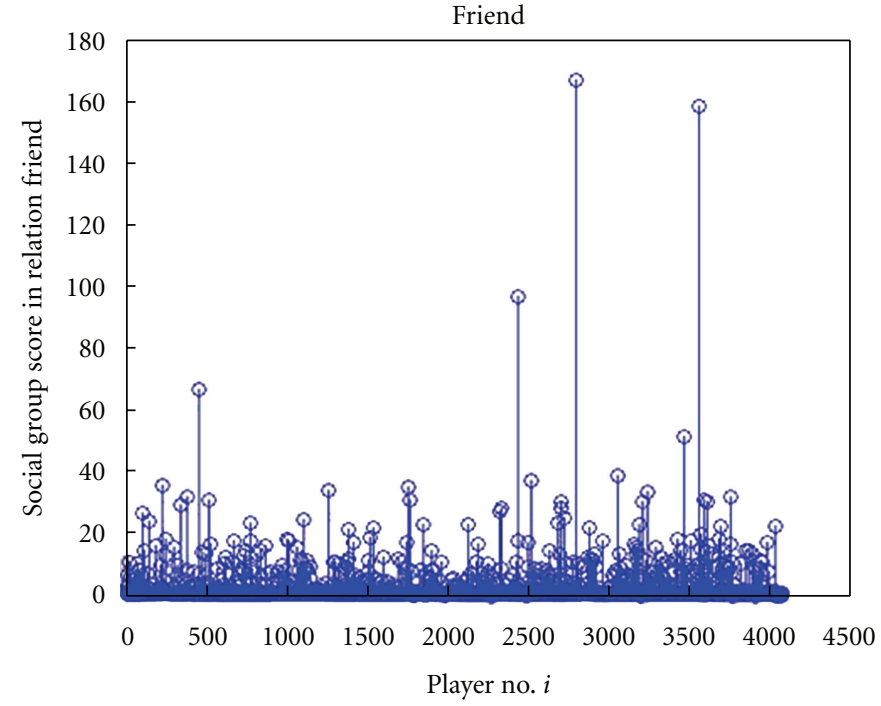

(b) Friend

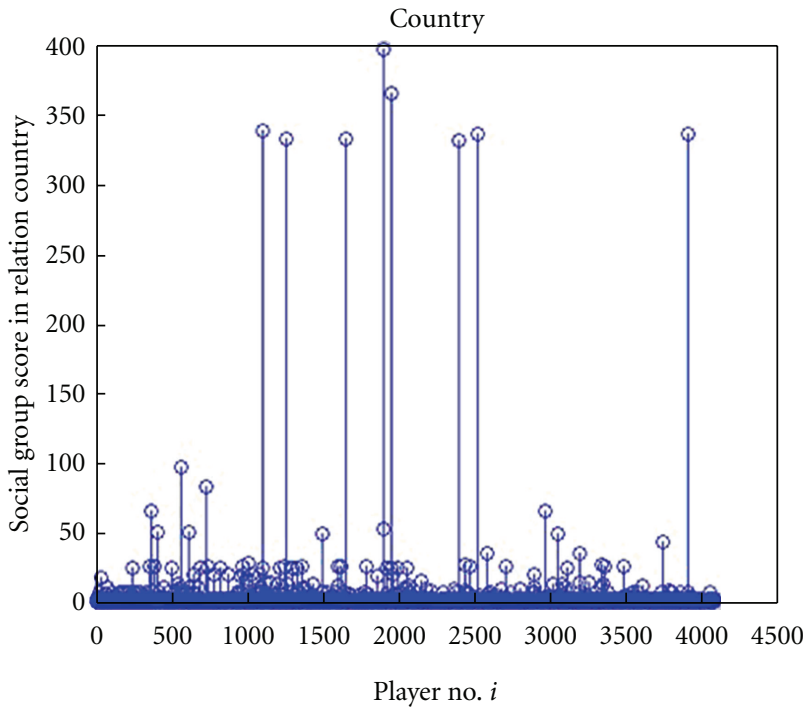

(c) Country

FIGURE 4: The $R_{i j}$ in the three relation groups.

TABle 3: The weight of different factors.

\begin{tabular}{lc}
\hline Category & Weight \\
\hline Player individual score & $65.68 \%$ \\
Couple relation social group score & $8.04 \%$ \\
Friend relation social group score & $13.52 \%$ \\
Country relation social group score & $12.76 \%$ \\
\hline
\end{tabular}

According to the player's relationship and achievement scores, players' categories can be classified by RA model [3]. Depending on the game design, the parameters $K$ of SN model will be different. The specific score is based on the game's operating experience.

With the features of "Empire Online" game design and players' data analyzing, we found that the attributes score
TABLE 4: Players distinguish table for $K$ value.

\begin{tabular}{lcc}
\hline Category & Range of $K$ & Player classification \\
\hline Type 1 & $K \in[0,0.2)$ & Achiever \\
Type 2 & $K \in[0.2,0.55)$ & Moderator \\
Type 3 & $K \in[0.55, \infty)$ & Socialize \\
\hline
\end{tabular}

of player have distinct taxonomy feature. When $K<0.2$, over $85 \%$ players have achievement motivation, it represents Achiever; when $K>0.55$, over $87 \%$ players have relationship motivation; it represents Socialize; the others are Moderator. It is appropriate to choose $K=0.2, K=0.55$ to distinguish the players' categories. It can be shown in the Table 4 . 


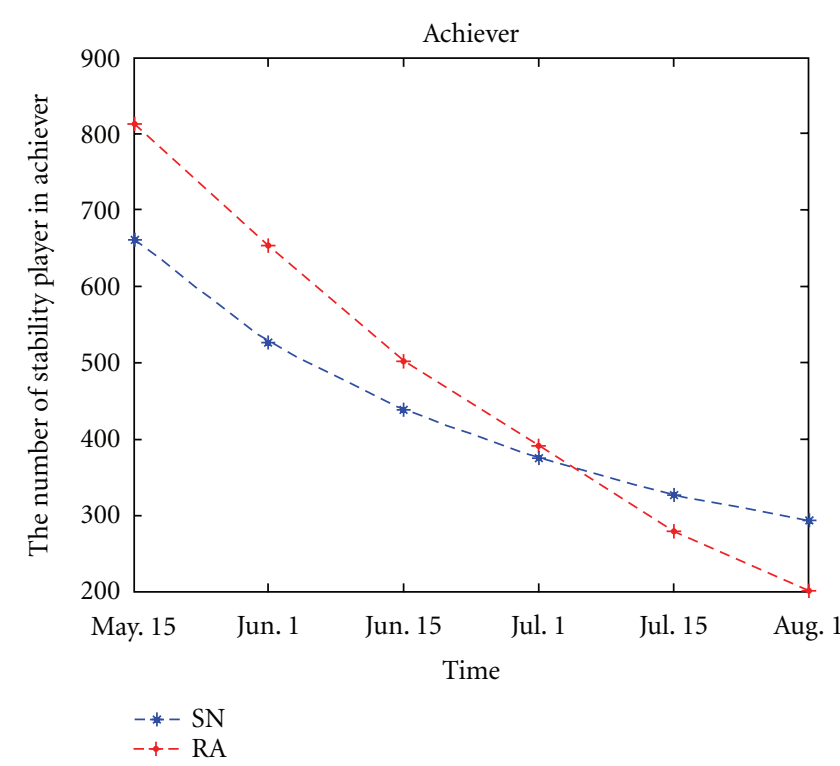

(a) Achiever

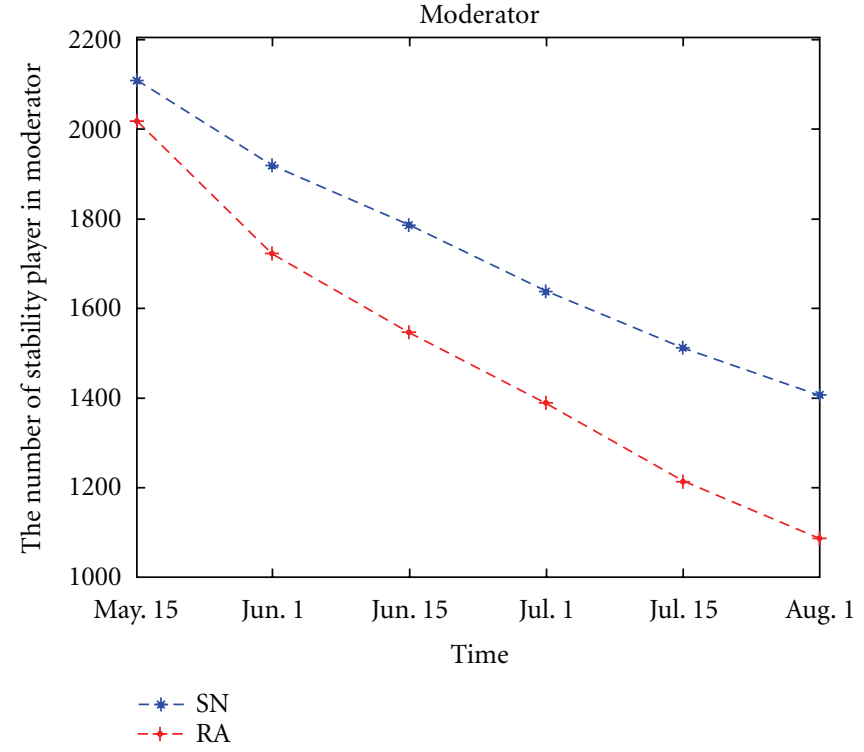

(b) Moderator

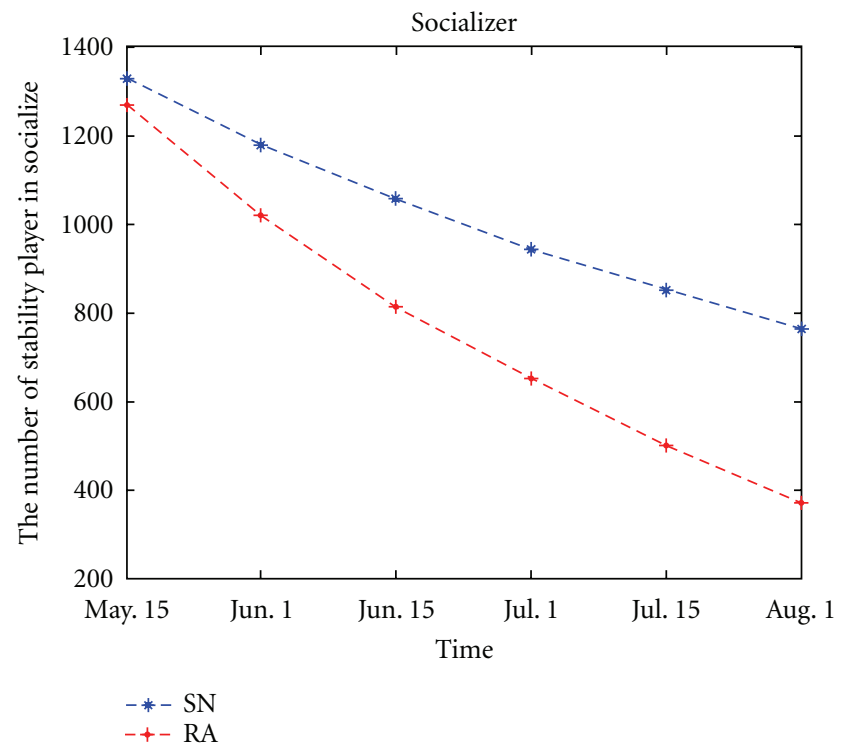

(c) Socializer

FigURE 5: The curve of stable player in three relation groups at different period.

4.2. The Comparison of the Stability between RA Model and SN Model. Through investigation, most subscribers are high school students and soldiers in China mobile MMOG. These categories people do not have enough time to expertise the game. Meanwhile, the mobile MMOG is relatively simple and the game content and version is not complex. The players often take months to experience the whole game version. Each year, the company has to update the new game version. In this way, the more stable of the player taxonomy model, the more useful for game design and monitor to balance the game world. These factors are very important for extending the game of the life.

In order to verify the usability and practicability of this model, we compare the stability of two models between RA
Model and SN Model. In the game, the taxonomy type of player is always changing (e.g., achiever to moderator and moderator to socialize). But the taxonomy category of player who has plentiful experience will be steady. If we survey the players' types at several different periods, we would get the number of changed players in each period. The players who keep in the same type are called stable players. If the model is more stable, the change of players' category is more inconspicuous. In this way, we can measure the usability and stability for SN and RA model.

In order to evaluate the model, the 4095 experienced players are selected. We use different models to calculate the taxonomy score every 15 days and record the number of stable players in each taxonomy category. As shown in the 
Figure 5, the number of unchanged players in each period of time and the result of these 3 categories of players. The $y$ axis indicates the number of stable players. The $x$-axis is the different period of time.

In the figure, three types of stability players' number in Moderator and Socialize have a downward trend. This is because we only calculated the number of the players whose type does not change in the period of time. As for the number of players who are from other type conversion, we have no statistics.

We can see that the downtrend and amplitude of curve means SN model is more stable than RA model and the result is also conforming to other three categories. The $\mathrm{SN}$ model can be proved as a better player's taxonomy model.

\section{Conclusion and Future Works}

In this paper, the impacts of social networks are mainly analyzed. By calculating players' own attribute score and social groups score, it is more accurate to quantize player's taxonomy. Using this model, we can know that not only players' own attributes, but also social groups have influence on players taxonomy, and then, we calculate the weights in the taxonomy. Also, we have proposed SN model which is based on social network, and it is applied to a commercial mobile game "Empire Online". It verifies that the social network have important impacts on taxonomy model. Further, the result shows that the SN model is more stable than RA model.

In the future, we will apply SN model to other mobile MMORPGs and continue our optimize study to SN model with other social networks analysis.

\section{Acknowledgments}

The work in this paper is in part supported by the National Natural Science Foundation of China under Grant nos. 61073132 and 60776796, the Natural Science Foundation of Guangdong Province of China under Grant no. 9151027501000035, Guangdong Province scientific and technological project under Grant no. 2009B010800017, and the Fundamental Research Funds for the Central Universities (101gpy33).

\section{References}

[1] R. Bartle, "Heats, clubs, diamonds, spades: players who suit MUDs," Journal of MUD Research, vol. 53, no. 1, pp. 86-107, 1996.

[2] P. Carrington, J. Scot, and S. Wasserman, Models and Methods in Social Network Analysis, Cambridge University Press, Cambridge, UK, 2005.

[3] F. You, J. Wang, Z. Zheng et al., "RA-model: a taxonomy model of player activities in mobile MMORPGs," in Proceedings of the 4th international conference on mobile technology, applications, and systems and the 1st international symposium on Computer human interaction in mobile technology (IS-CHI '07), pp. 704707, September 2007.

[4] N. Yee, "The demographics, motivations, and derived experiences of users of massively multi-user online graphical environments," Presence, vol. 15, no. 3, pp. 309-329, 2006.
[5] N. Yee, "Motivations of play in online games," Journal of CyberPsychology and Behavior, vol. 9, pp. 772-775, 2007.

[6] E. Castronova, Synthetic Worlds: The Business and Culture of Online Games, University of Chicago Press, Chicago, Ill, USA, 2005.

[7] N. Ducheneaut and R. J. Moore, "The social side of gaming: a study of interaction patterns in a massively multiplayer online game," in Proceedings of the Computer Supported Cooperative Work (CSCW'04), pp. 360-369, November 2004.

[8] N. Ducheneaut, R. J. Moore, and E. Nickell, "Virtual "third places": a case study of sociability in massively multiplayer games," Computer Supported Cooperative Work, vol. 16, no. 12, pp. 129-166, 2007.

[9] N. Ducheneaut, N. Yee, E. Nickell, and R. J. Moore, "Alone together?' Exploring the social dynamics of massively multiplayer online games," in Proceedings of the Conference on Human Factors in Computing Systems (CHI '06), pp. 407-416, April 2006.

[10] M. Jakobson and T. L. Taylor, "The sopranos meets everquest: social networking in massively multiplayer online games," in Proceedings of the Design Automation Conference (DAC '3), pp. 81-90, 2003.

[11] N. Ducheneaut, N. Yee, E. Nickell, and R. J. Moore, "The life and death of online gaming communities: a look at guilds in world of warcraft," in Proceedings of the 25th Conference on Human Factors in Computing Systems (CHI '07), pp. 839-848, May 2007.

[12] C. Bo, R. Kraut, and M. Fichman, Matching People and Groups: Recruitment and Selection in Online Games, Carnegie Mellon University, 2008.

[13] M. Granovetter, “The strength of weak ties," American Journal of Economics and Sociology, vol. 78, pp. 1360-1380, 1973.

[14] F. Harary, "On the notion of balance of signed graph," Michigan Mathematical Journal, vol. 2, pp. 143-146, 1953.

[15] P. Doreian and A. Mrvar, "A partitioning approach to structural balance," Social Networks, vol. 18, no. 2, pp. 149-168, 1996.

[16] B. Schneider, "The people make the place," Personnel Psychology, vol. 40, pp. 437-453, 1987.

[17] B. Schneider, D. B. Smith, and H. W. Goldstein, "Attractionselection-attrition: toward a person-environment psychology of organizations," in Person-Environment Psychology: New Directions and Perspectives, B. W. Walsh, K. H. Craik, and R. H. Price, Eds., pp. 61-86, Erlbaum, Mahway, NJ, USA, 2nd edition, 2000.

[18] D. Williams, N. Martins, M. Consalvo, and J. D. Ivory, "The virtual census: representations of gender, race and age in video games," New Media and Society, vol. 11, no. 5, pp. 815-834, 2009.

[19] N. F. Johnson, C. Xu, Z. Zhao et al., "Human group formation in online guilds and offline gangs driven by a common team dynamic," Physical Review E, vol. 79, no. 6, Article ID 066117, 11 pages, 2009.

[20] M. Szell and S. Thurner, "Measuring social dynamics in a massive multiplayer online game," Social Networks, vol. 32, no. 4, pp. 313-329, 2010. 

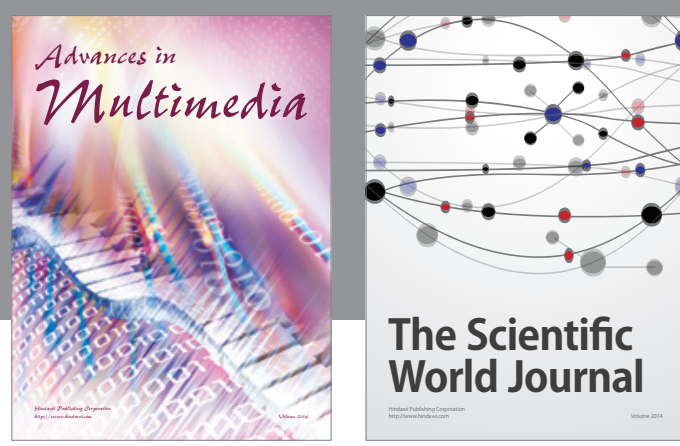

The Scientific World Journal
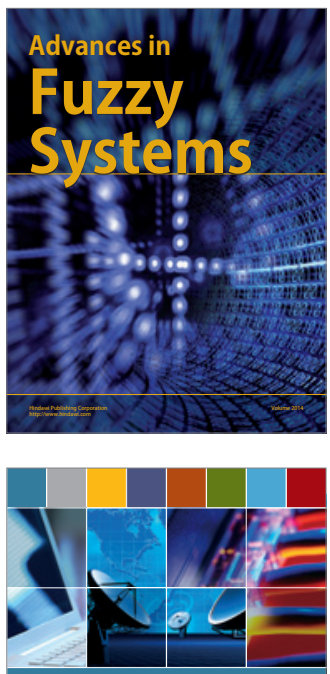

Computer Networks and Communications
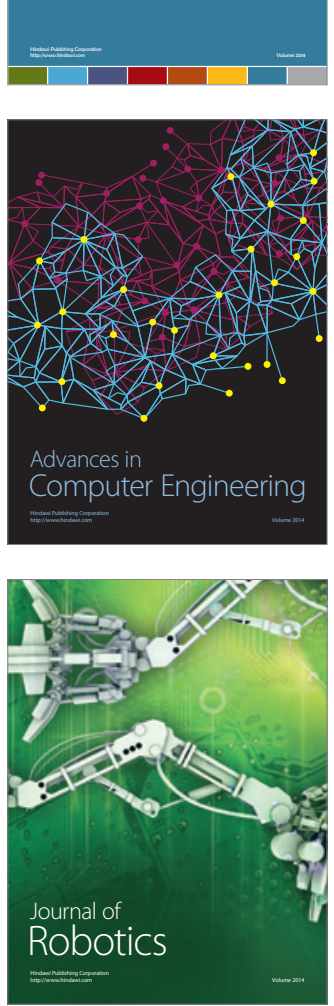
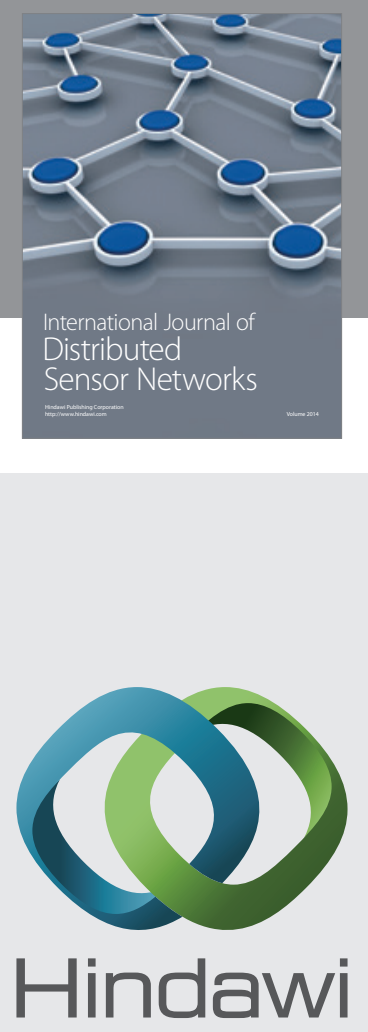

Submit your manuscripts at

http://www.hindawi.com
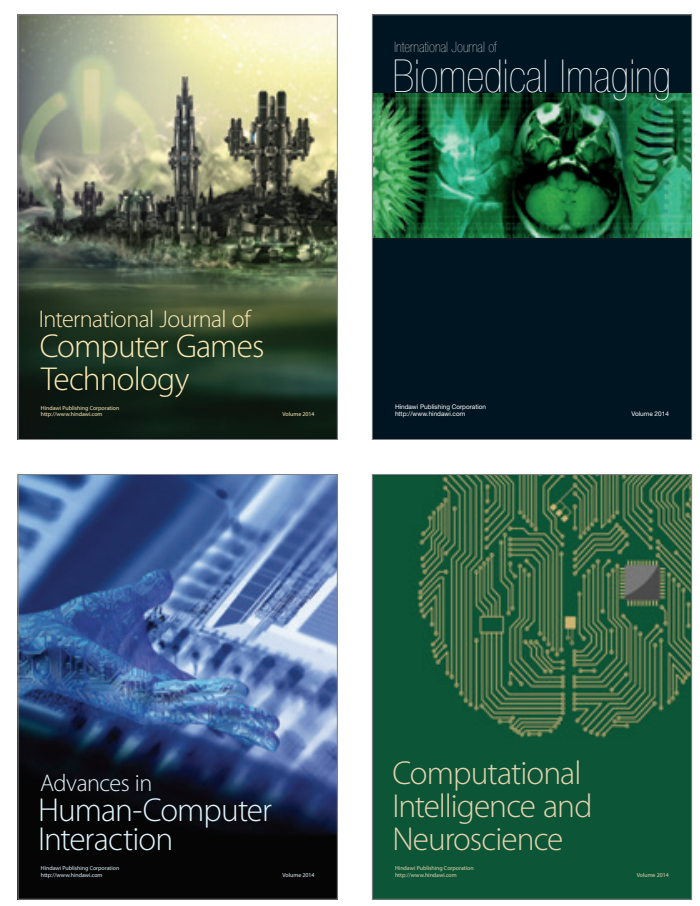
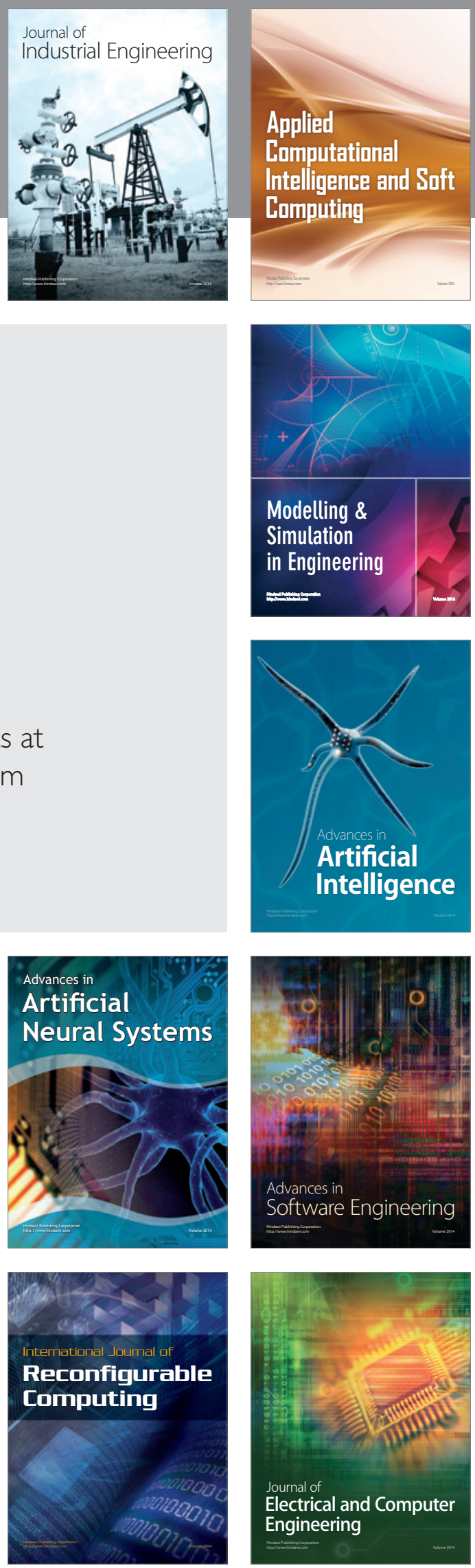